\title{
Efficacy of Ultrasound-Guided Core Needle Biopsy in Detecting Metastatic Axillary Lymph Nodes in Breast Cancer
}

\author{
Jeeyeon Lee, Ho Yong Park, Wan Wook Kim, Chan Sub Park, Minhye Jeong, Jin Hyang Jung \\ Department of Surgery, Kyungpook National University Chilgok Hospital, School of Medicine, Kyungpook National University, \\ Daegu, Korea
}

Received August 12, 2020

Revised October 8, 2020

Accepted October 14, 2020

\section{Correspondence to:}

Jin Hyang Jung

Department of Surgery, Kyungpook

National University Chilgok

Hospital, School of Medicine,

Kyungpook National University,

807 Hoguk-ro, Buk-gu, Daegu

41404, Korea

Tel: $+82-53-200-2703$

Fax: $+82-53-200-2072$

E-mail: jjh01@knu.ac.kr
Purpose: Axillary lymph node status is an important prognostic factor in breast cancer. Axillary lymph nodes can be evaluated using fine-needle aspiration cytology (FNAC) or core needle biopsy (CNB) before surgery. This study compared the accuracy and false-negative rates between FNAC and CNB in patients with breast cancer who either did or did not receive neoadjuvant chemotherapy (NAC).

Methods: The clinicopathological factors of the patients were analyzed retrospectively, and the sensitivity, specificity, positive and negative predictive values, false-positive and false-negative rates, and accuracy of FNAC $(n=27)$ and CNB $(n=23)$ were compared.

Results: Regardless of whether or not NAC was performed, the CNB evaluation of the metastatic axillary lymph nodes had a $100.0 \%$ sensitivity, specificity, negative predictive value, and accuracy, except for one case with an inadequate sample. In the FNAC group, the false-negative rate was higher in patients with breast cancer who received NAC before evaluating the lymph nodes $(9.1 \%$ vs. $7.7 \%)$. Moreover, ultrasound imaging was the most sensitive imaging modality that can detect the suspicious axillary lymph node.

Conclusion: $\mathrm{CNB}$ was more effective in evaluating the axillary lymph nodes in breast cancer than FNAC and was performed without major complications.

Keywords: Axillary lymph nodes, Biopsy, Breast cancer, Cytology

\section{INTRODUCTION}

Axillary staging in breast cancer is an important prognostic factor, and the standard evaluation for axillary lymph nodes would require full dissection. $(1,2)$ However, axillary lymph node dissection (ALND) causes various sequelae, including numbness, pain, impaired arm movement, and lymphedema.(3,4) Although sentinel lymph node biopsy (SLNB) could be an alternative procedure for evaluating axillary lymph nodes in breast cancer, partic- ularly for early breast cancer or clinically node-negative breast cancer, $(5,6)$ it also presents with a possibility of such morbidities, albeit with a lower incidence rate. Therefore, several clinical trials on the omission of axillary staging in early breast cancer have been conducted. $(7,8)$

The pathologic assessment of axillary lymph nodes is usually performed with fine needle aspiration cytology (FNAC) or core needle biopsy (CNB).(9-11) Although the false-negative rate of FNAC for axillary lymph nodes in breast cancer is reported to be relatively high at $10 \%-$ $31 \%$,(12-14) FNAC is more frequently used than CNB for 
axillary evaluation due to its lower incidence of procedural complications. However, FNAC cannot replace the surgical assessment of axillary lymph nodes. Moreover, CNB for evaluating axillary lymph nodes has shown high accuracy and low false-negative rates. $(10,11,15)$

The aim of this study is to compare the accuracy and false-negative rates between FNAC and CNB for evaluating axillary lymph nodes in patients with breast cancer who did and did not receive neoadjuvant chemotherapy.

\section{METHODS}

Between 2011 and 2016, 159 patients with cN1 breast cancer underwent surgery and additional treatments at the Kyungpook National University Hospital. Among them, 74 patients who underwent a pathologic evaluation for suspicious axillary lymph nodes before surgery were included in this study. This pathologic evaluation was performed using FNAC or CNB. We excluded 24 cases which showed conversion to radiologic complete response (rCR) after neoadjuvant chemotherapy. Furthermore, the FNAC and CNB groups were compared based on various clinicopathologic factors in this study.

Clinical records and pathologic results were all reviewed and analyzed retrospectively. Moreover, the clinicopathologic variables included patient age at breast cancer diagnosis, body mass index, clinical and pathologic tumor size, clinical lymph node status, neoadjuvant or adjuvant treatment performance, and oncologic outcomes. If the patients received neoadjuvant chemotherapy (NAC) before surgery, the rCR for breast lesions and axillary lymph nodes was also evaluated using mammography, ultrasonography, breast magnetic resonance imaging (MRI), and chest/abdominal computed tomography (CT).

The breast cancers were classified into four different molecular subtypes based on their immunohistochemistry (IHC) biomarkers: estrogen receptor, progesterone receptor (PR), HER2 gene status, and the Ki67 index. The Ki67 proliferation index was considered high when $>15 \%$ of tumor cells showed nuclear immunoreactivity. The his- topathological examination of the four biomarkers was performed in accordance with the criteria in the ASCO/CAP (American Society of Clinical Oncology/ College of American Pathologists) 2016 guidelines.

The FNAC procedures were performed under ultrasonography guidance; each lesion was aspirated with a 21-gauge needle using the to-and-fro method. Each aspirated material was smeared on the glass slides and was immediately fixed in 95\% alcohol. All FNAC slides were prepared for Papanicolaou staining in accordance with the standard method. Moreover, the CNB procedures were performed under ultrasonography guidance using 18-gauge cutting needle biopsy instruments (Stericut $^{\circledR}$, TSK Laboratory, Hirayanagi-Cho, Japan). At least two core samples including cortical tissue were obtained from suspicious axillary lymph nodes. The CNB samples were immediately fixed with $10 \%$ neutral buffered formalin and then paraffin-embedded. The CNB specimens were prepared and stained with hematoxylin and eosin. Moreover, the diagnosis of FNAC and CNB was confirmed by a cytopathologist with comparable experience.

All procedures in this study that involved human participants were performed in accordance with the ethical standards of the institutional review board of Kyungpook National Hospital, and the experimental protocol was also approved by them (KNUCH 2015-05-205). Furthermore, all the experiments were performed in accordance with the relevant guidelines and regulations.

\section{Surgical methods}

All patients underwent SLNB or ALND depending on the condition of the axillary lymph nodes. To identify the sentinel lymph node radiolabeled colloid, blue dye, or a combination of these methods was used. A gamma probe identified radioactivity in the lymph nodes in the axilla, and the surgeons visually identified the blue-stained lymphatic nodes. Moreover, the axilla was meticulously examined and any palpable or visually abnormal lymph nodes were resected and submitted for frozen biopsy. Conventional ALND included the removal of level I and II axillary lymph nodes. 


\section{Statistical analysis}

To assess the impact of the CNB procedure for suspicious axillary lymph nodes, the sensitivity, specificity, positive and negative predictive values, false-positive and false-negative rates, and accuracy rates of FNAC and CNB were calculated and compared. The detection rates of suspicious axillary lymph nodes in ultrasound, chest CT, and breast MRI were also evaluated.

All statistical analyses were performed using SPSS ver. 25.0 (SPSS, Chicago, IL, USA). Categorical variables were analyzed using the chi-squared test through univariate analysis. A P-value $<0.05$ was considered as statistically significant.

\section{RESULTS}

No statistically significant differences were observed between the patients who received FNAC $(n=27)$ and CNB ( = 23) with regard to the mean age and body mass index, type of breast, and axillary surgery, mean clinical and pathologic tumor size, and molecular biomarkers. During the mean follow-up period of 55.2 months ( $47.3 \pm 16.8$ in FNAC vs. $58.2 \pm 78.4$ in CNB), five cases of locoregional recurrence ( 2 in FNAC vs. 3 in CNB, $P=0.518$ ), two cases of distant metastasis ( 0 in FNAC vs. 2 in $C N B, P=0.123$ ), and one case of death in the FNAC group were observed. Moreover, after the procedure, only one case of hematoma was detected in the CNB group ( $\mathrm{P}=0.941$; Table 1 ).

In 17 patients who underwent neoadjuvant chemotherapy (NAC), the FNAC and CNB procedures were per-

Table 1. Clinicopathologic Factors of Patients with Breast Cancer Who Were Evaluated for Suspicious Axillary Lymph Nodes Using Fine Needle Aspiration Cytology and Core Needle Biopsy

\begin{tabular}{|c|c|c|c|}
\hline Variables & Fine needle aspiration cytology $(\mathrm{n}=27)$ & Core needle biopsy $(\mathrm{n}=23)$ & P-value \\
\hline Mean patient's age (years, \pm SD) & $54.0 \pm 11.2$ & $49.7 \pm 15.9$ & 0.274 \\
\hline Body mass index $\left(\mathrm{kg} / \mathrm{m}^{2}, \pm \mathrm{SD}\right)$ & $24.7 \pm 3.0$ & $23.3 \pm 2.6$ & 0.106 \\
\hline \multicolumn{4}{|l|}{ Breast surgery $(n, \%)$} \\
\hline Breast conserving surgery & $17(63.0)$ & $15(65.2)$ & 0.540 \\
\hline Mastectomy & $10(37.0)$ & $8(34.8)$ & 0.695 \\
\hline \multicolumn{4}{|l|}{ Axillary surgery (n, \%) } \\
\hline Sentinel lymph node biopsy & $17(63.0)$ & $13(56.5)$ & 0.651 \\
\hline Axillary lymph node dissection & $10(37.0)$ & $10(43.48)$ & 0.407 \\
\hline Mean clinical tumor size $(\mathrm{cm}, \pm \mathrm{SD})$ & $4.3 \pm 3.2$ & $3.3 \pm 1.9$ & 0.074 \\
\hline Mean pathologic tumor size $(\mathrm{cm}, \pm \mathrm{SD})$ & $2.8 \pm 2.6$ & $2.3 \pm 1.5$ & 0.435 \\
\hline Estrogen receptor, positive (n, \%) & $16(55.6)$ & $17(73.9)$ & 0.114 \\
\hline Progesterone receptor, positive (n, \%) & $12(44.5)$ & $16(69.6)$ & 0.077 \\
\hline HER2 gene expression, positive ( $n, \%)$ & $8(28.6)$ & $5(21.7)$ & 0.157 \\
\hline Triple-negative breast cancer (n, \%) & $5(18.5)$ & $3(13.0)$ & 0.607 \\
\hline \multicolumn{4}{|l|}{ Ki67 index (n, \%) } \\
\hline$<15 \%$ & $5(22.7)$ & $6(26.1)$ & 0.460 \\
\hline$\geq 15 \%$ & $16(72.7)$ & $16(69.6)$ & 0.095 \\
\hline Neoadjuvant chemotherapy (n, \%) & $12(44.5)$ & $6(26.1)$ & 0.632 \\
\hline Adjuvant chemotherapy (n, \%) & $17(63.0)$ & $16(69.6)$ & 0.920 \\
\hline Adjuvant radiotherapy $(\mathrm{n}, \%)$ & $22(81.5)$ & $19(82.6)$ & 0.418 \\
\hline Adjuvant hormone treatment $(\mathrm{n}, \%)$ & $17(63.0)$ & $17(73.9)$ & 0.415 \\
\hline Mean follow-up period (months, \pm SD) & $47.3 \pm 16.8$ & $58.2 \pm 78.4$ & 0.501 \\
\hline Locoregional recurrence (n, \%) & $2(7.4)$ & $3(13.0)$ & 0.518 \\
\hline Distant metastasis (n, \%) & 0 & $2(8.7)$ & 0.123 \\
\hline Death $(n, \%)$ & $1(3.7)$ & 0 & 0.352 \\
\hline Post-procedural complication (hematoma) (n, \%) & 0 & $1(4.3)$ & 0.941 \\
\hline
\end{tabular}

$\mathrm{SD}=$ standard deviation, HER2 = human epidermal growth factor receptor 2. 
formed in 12 and 5 cases, respectively. Moreover, in 33 patients who did not receive NAC, the FNAC, and CNB procedures were performed in 15 and 18 cases, respectively, to evaluate the suspected metastasis to axillary lymph nodes, respectively. Four cases of technical failure were observed, including procedural failure and surgical failure. Two cases in FNAC showed cell counts that were too small to assess the metastasis of breast cancer, and one case in CNB showed only fibrofatty tissue in cores. In one case of surgical failure, ALND had to be performed due to the undetectable malignancy in the lymph nodes or sentinel lymph nodes. Therefore, the accurate pathologic findings of the malignancy-proven lymph nodes could not be recognized (Fig. 1).
Regardless of performing NAC, CNB for the evaluation of metastatic axillary lymph node showed better results than FNAC in terms of sensitivity, specificity, negative predictive value, false-negative rates, and accuracy. In the FNAC group, the false-negative rate was higher in patients with breast cancer who received NAC before lymph node evaluation (Table 2).

Among the imaging modalities, including axillary ultrasound, chest CT, and breast MRI, the axillary ultrasound showed superior detection rates compared to chest CT and breast MRI in both non-malignant and malignant lymph node groups (Table 3).

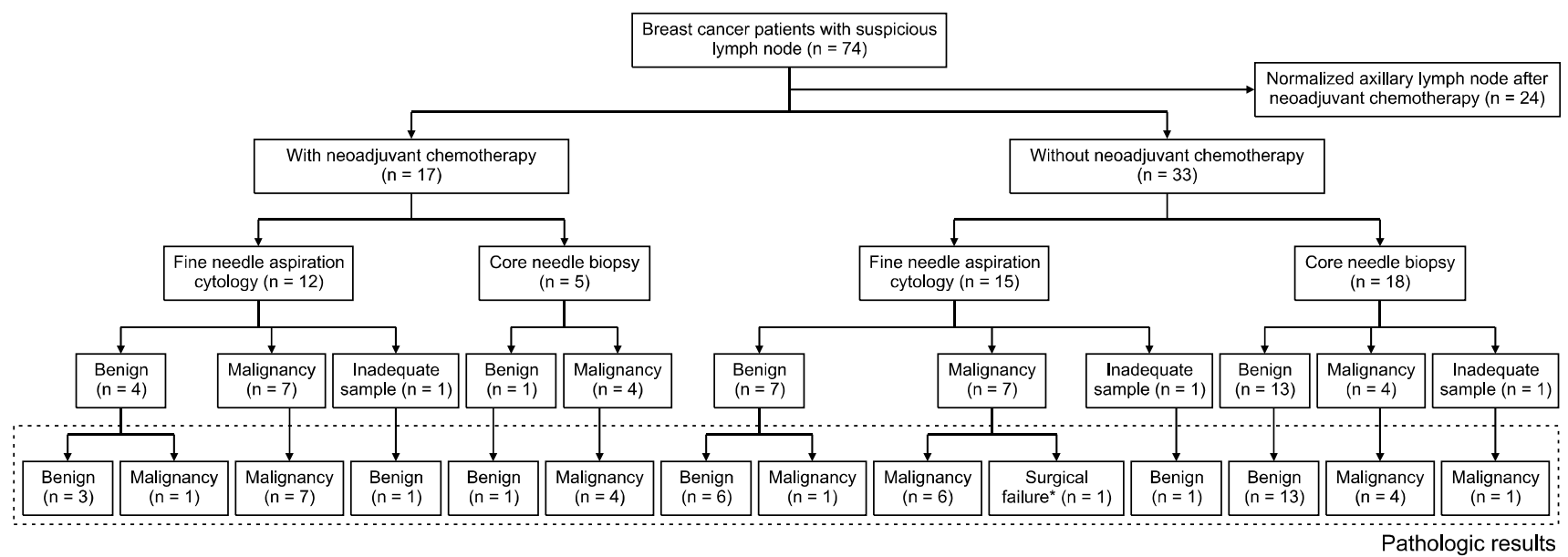

Fig. 1. Schematic correlation diagram between fine needle aspiration cytology, core needle biopsy, and pathologic results.

Table 2. Statistical Quality Criteria of Fine-Needle Aspiration Cytology and Core Needle Biopsy for Evaluation of Suspicious Axillary Lymph Node*

\begin{tabular}{lcccrr}
\hline \multirow{2}{*}{ Statistical quality criteria $(\mathrm{n}, \%)$} & \multicolumn{2}{c}{ Fine needle aspiration cytology $(\mathrm{n}=27)$} & & \multicolumn{2}{c}{ Core needle biopsy $(\mathrm{n}=23)$} \\
\cline { 2 - 3 } \cline { 5 - 6 } & With NAC $(\mathrm{n}=11)$ & Without NAC $(\mathrm{n}=13)$ & & With NAC $(\mathrm{n}=5)$ & Without NAC $(\mathrm{n}=17)$ \\
\hline Sensitivity & $7 / 8(87.5)$ & $6 / 7(85.7)$ & $6 / 6(100.0)$ & $4 / 4(100.0)$ & $4 / 4(100.0)$ \\
Specificity & $3 / 3(100.0)$ & $6 / 6(100.0)$ & $1 / 1(100.0)$ & $13 / 13(100.0)$ \\
Positive predictive value & $7 / 7(100.0)$ & $6 / 7(85.7)$ & 0 & $4 / 4(100.0)$ & $4 / 4(100.0)$ \\
Negative predictive value & $3 / 4(75.0)$ & 0 & $1 / 13(7.7)$ & 0 & $13 / 13(100.0)$ \\
False-positive & $1 / 11(9.1)$ & $12 / 13(92.3)$ & 0 & 0 \\
False-negative & $10 / 11(90.9)$ & $5 / 5(100.0)$ & $17 / 17(100.0)$ \\
Accuracy & & & & & 0 \\
\hline
\end{tabular}

*Three cases of inadequate sample and one case of failure in identifying lymph node were excluded.

$\mathrm{NAC}=$ neoadjuvant chemotherapy. 
Table 3. Detection Rate in Ultrasound, Chest CT, and Breast MRI of Suspicious Axillary Lymph Nodes Based on Pathologic Results*

\begin{tabular}{|c|c|c|c|c|c|c|c|c|}
\hline \multirow{2}{*}{ Pathologic results (n, \%) } & \multicolumn{4}{|c|}{ Fine needle aspiration cytology $(n=26)$} & \multicolumn{4}{|c|}{ Core needle biopsy $(\mathrm{n}=23)$} \\
\hline & Ultrasound & CT & MRI & Total & Ultrasound & CT & MRI & Total \\
\hline Non-malignant lymph node & $9(81.8)$ & $8(72.7)$ & $8(72.7)$ & 11 & $13(92.9)$ & $10(71.4)$ & $5(35.7)$ & 14 \\
\hline Malignant lymph node & $15(100.0)$ & $14(93.3)$ & $10(66.7)$ & 15 & $9(100.0)$ & $6(66.7)$ & $8(88.9)$ & 9 \\
\hline
\end{tabular}

*One case of failure in identifying the accurate lymph node was excluded.

$\mathrm{CT}=$ computational tomography; MRI = magnetic resonance imaging.

\section{DISCUSSION}

Over the past decades, the cases of breast cancer requiring surgical treatment have declined. In breast cancer surgery, the development of radiotherapy as an adjuvant treatment has enabled breast conservation, and it has been reported that nipple-sparing mastectomy is not inferior to conventional mastectomy.(16-20)

Even the need for axillary surgery has declined. The concept of SLNB, which is still considered a revolutionary surgical concept, could be applied for axillary staging instead of ALND, which has been reported to greatly reduce postoperative morbidities, such as postoperative seroma, numbness, axillary pain, limitation of arm movement, and lymphedema.(3-5) In 2014 and 2018, the EORTC (European Organisation for Research and Treatment of Cancer) has reported that axillary radiotherapy is not inferior to ALND in the treatment of metastatic axillary lymph nodes in breast cancer (AMAROS study), even after undergoing NAC. $(21,22)$ During the similar period, the US Alliance Group had reported the possibility of the use of SLNB for selected patients who received NAC based on the results of a 10-year long-term follow-up indicating that SLNB was not inferior to ALND in patients with less than three metastatic axillary lymph nodes after NAC. $(23,24)$

Recently, the EIO (European Institute of Oncology) had initiated the SOUND (Sentinel node vs. Observation after axillary Ultra-souND) trial which was designed to compare SLNB with only surveillance with axillary ultrasound in node-negative early breast cancer.(7) Moreover, the most important evidence that supports the omission of axillary staging is that removing the axillary lymph nodes does not seem to have a direct impact on the actual overall survival. However, to skip axillary surgery, an alternative procedure should be used for axillary staging to predict the prognosis of breast cancer. Although axillary ultrasound may be sufficient to replace SLNB, even if the result of the SOUND trial has not been officially reported yet, an alternative method of tissue confirmation is necessary to omit surgical evaluation in advanced breast cancer and NAC-treated breast cancer cases.

Generally, the pathologic assessment for axillary lymph nodes is performed with FNAC or CNB.(9-11) Because FNAC is easier and less invasive, it is more frequently performed than CNB. However, because its false-negative rate can be as high as $58 \%$, surgical evaluation is necessary.(12) Conversely, the false-negative rate of $\mathrm{CNB}$ is much lower at $0 \%-14 \%$. The ultrasound-guided CNB in particular shows better results compared to ultrasound-guided FNA in evaluating axillary lymph nodes in breast cancer after NAC. $(12,15,25-27)$

Metastasis to axillary lymph nodes is easily detected using various imaging modalities. The accuracy of ultrasound in detecting axillary lymph node metastasis in breast cancer is reportedly $67 \%-96 \%$, and the results further improve greatly if the breast cancer was previously treated with NAC. $(28,29)$ The sensitivity and specificity of breast MRI for evaluating axillary lymph nodes in breast cancer are reportedly $33.3 \%-97.0 \%$ and $14.0 \%-98.5 \%$, respectively. (29-32) In the present study, although the suspicious axillary lymph node was the most frequently detected with ultrasound imaging, the false-positive rate was also the highest with ultrasound imaging. Therefore, pathologic confirmation is necessary to evaluate metastasis to axillary 
lymph nodes in breast cancer. Improved results in the identification of metastatic axillary lymph node may provide the evidence supporting the omission of axillary surgical staging.

Moreover, because the lesions showing locoregional recurrence or distant metastasis may not be biologically equivalent to primary tumor, tissue biopsy is necessary for not only an accurate diagnosis of the lesion but also the assessment of biologic features. When the recurrence is only detected in axillary lymph nodes because only the existence of malignant cells can be detected with FNAC, CNB is necessary to provide biologic information in order to develop a treatment strategy.

Although several studies have reported a significantly higher occurrence of post-procedural complications, such as hematoma or bruising, in CNB compared to FNA, (10,26,33-35) most complications can be managed with only conservative treatment. In a recent study, only one case presented with hematoma after $\mathrm{CNB}$, and this complication was resolved spontaneously within a week.

Although this retrospective study did not have a large population of breast cancer patients and did not involve any randomization of procedures, we believe that the efficacy of CNB in the assessment of axillary lymph nodes has been sufficiently evaluated.

\section{CONCLUSION}

Overall, CNB is more effective than FNAC in evaluating axillary lymph nodes in breast cancer and can be performed without major complications. Because the tissue biopsy can provide information on not only the diagnosis of lesion but also its biologic features, ultrasound-guided CNB for axillary lymph nodes can be useful in examining lymph nodes with metastasis recurrence as well in cases wherein SLNB has to be omitted.

\section{CONFLICT OF INTEREST}

The authors declare that they have no conflict of interests.

\section{DATA AVAILABILITY}

The datasets generated and/or analyzed in this study are not publicly available. However, they can be obtained from the corresponding author upon reasonable request.

\section{COMPLIANCE WITH ETHICAL STANDARDS}

In this study, all procedures that involved human participants were performed in accordance with the ethical standards of the Institutional Review Board of Kyungpook National University Chilgok Hospital (KNUCH 2020-03009) and the 1964 Helsinki declaration and its later amendments or comparable ethical standards.

Informed consent was obtained from all individual participants included in the study.

\section{ACKNOWLEDGEMENTS}

This work was supported by the National Research Foundation of Korea (NRF) grant funded by the Korea government (2014R1A5A2009242, 2019R1F1A1063853) and by a grant from the National R\&D Program for Cancer Control, Ministry of Health and Welfare, Republic of Korea (1420040). And this research was supported by a grant of the Korea Health Technology R\&D Project through the Korea Health Industry Development Institute (KHIDI), funded by the Ministry of Health \& Welfare, Republic of Korea (HI17C1142). This work was supported by the National Research Foundation of Korea (NRF) grant funded by the Korea government (MSIT) (NRF-2019R1A2C1006264). This work was supported by the National Research Foundation of Korea (NRF) grant funded by the Korea government (2017M3A9G8083382).

\section{REFERENCES}

1. Reynolds C, Mick R, Donohue JH, Grant CS, Farley DR, Callans LS, et al. Sentinel lymph node biopsy with metastasis: can axil- 
lary dissection be avoided in some patients with breast cancer? J Clin Oncol 1999;17:1720-6.

2. Lovrics PJ, Chen V, Coates G, Cornacchi SD, Goldsmith CH, Law $\mathrm{C}$, et al. A prospective evaluation of positron emission tomography scanning, sentinel lymph node biopsy, and standard axillary dissection for axillary staging in patients with early stage breast cancer. Ann Surg Oncol 2004;11:846-53.

3. Langer I, Guller U, Berclaz G, Koechli OR, Schaer G, Fehr MK, et al. Morbidity of sentinel lymph node biopsy (SLN) alone versus SLN and completion axillary lymph node dissection after breast cancer surgery: a prospective Swiss multicenter study on 659 patients. Ann Surg 2007;245:452-61.

4. Veronesi U, Paganelli G, Viale G, Luini A, Zurrida S, Galimberti $\mathrm{V}$, et al. A randomized comparison of sentinel-node biopsy with routine axillary dissection in breast cancer. N Engl J Med 2003;349:546-53.

5. Gipponi M, Bassetti C, Canavese G, Catturich A, Di Somma C, Vecchio C, et al. Sentinel lymph node as a new marker for therapeutic planning in breast cancer patients. J Surg Oncol 2004;85:102-11.

6. Sanuki N, Takeda A, Amemiya A, Ofuchi T, Ono M, Ogata H, et al. Outcomes of clinically node-negative breast cancer without axillary dissection: can preserved axilla be safely treated with radiation after a positive sentinel node biopsy? Clin Breast Cancer 2013;13:69-76.

7. Gentilini O, Veronesi U. Abandoning sentinel lymph node biopsy in early breast cancer? A new trial in progress at the European Institute of Oncology of Milan (SOUND: Sentinel node vs Observation after axillary UltraSouND). Breast 2012;21:678-81.

8. van Roozendaal LM, Vane MLG, van Dalen T, van der Hage JA, Strobbe LJA, Boersma LJ, et al. Clinically node negative breast cancer patients undergoing breast conserving therapy, sentinel lymph node procedure versus follow-up: a Dutch randomized controlled multicentre trial (BOOG 2013-08). BMC Cancer 2017:17:459.

9. Yu YH, Mo QG, Zhu X, Gao LQ, Liang C, Huang Z, et al. Axillary fine needle aspiration cytology is a sensitive and highly specific technique for the detection of axillary lymph node metastasis: a meta-analysis and systematic review. Cytopathology 2016;27: 59-69.

10. Rautiainen S, Masarwah A, Sudah M, Sutela A, Pelkonen O, Joukainen S, et al. Axillary lymph node biopsy in newly diagnosed invasive breast cancer: comparative accuracy of fine-needle aspiration biopsy versus core-needle biopsy. Radiology 2013;269:54-60.

11. Bhandari A, Xia E, Wang Y, Sindan N, Kc R, Guan Y, et al. Impact of sentinel lymph node biopsy in newly diagnosed invasive breast cancer patients with suspicious node: a comparative accuracy survey of fine-needle aspiration biopsy versus core-needle biopsy. Am J Transl Res 2018;10:1860-73.

12. Kane G, Fleming C, Heneghan H, McCartan D, James P, Trueick R, et al. False-negative rate of ultrasound-guided fine-needle aspiration cytology for identifying axillary lymph node metastasis in breast cancer patients. Breast J 2019;25: 848-52.

13. Ewing DE, Layfield LJ, Joshi CL, Travis MD. Determinants of false-negative fine-needle aspirates of axillary lymph nodes in women with breast cancer: lymph node size, cortical thickness and hilar fat retention. Acta Cytol 2015;59:311-4.

14. Wang Y, Dong H, Wu H, Zhang L, Yuan K, Chen H, et al. Improved false negative rate of axillary status using sentinel lymph node biopsy and ultrasound-suspicious lymph node sampling in patients with early breast cancer. BMC Cancer 2015;15:382.

15. Vidya R, Iqbal FM, Bickley B. Pre-operative axillary staging: should core biopsy be preferred to fine needle aspiration $\mathrm{cy}^{-}$ tology? Ecancermedicalscience 2017;11:724.

16. Fisher B, Redmond C, Poisson R, Margolese R, Wolmark N, Wickerham L, et al. Eight-year results of a randomized clinical trial comparing total mastectomy and lumpectomy with or without irradiation in the treatment of breast cancer. $\mathrm{N}$ Engl J Med 1989;320:822-8.

17. Fisher B, Anderson S, Bryant J, Margolese RG, Deutsch M, Fisher ER, et al. Twenty-year follow-up of a randomized trial comparing total mastectomy, lumpectomy, and lumpectomy plus irradiation for the treatment of invasive breast cancer. N Engl J Med 2002;347:1233-41.

18. Huang J, Mo Q, Zhuang Y, Qin Q, Huang Z, Mo J, et al. Oncological safety of nipple-sparing mastectomy in young patients with breast cancer compared with conventional mastectomy. Oncol Lett 2018;15:4813-20.

19. Caruso F, Ferrara M, Castiglione G, Trombetta G, De Meo L, Catanuto G, et al. Nipple sparing subcutaneous mastectomy: sixty-six months follow-up. Eur J Surg Oncol 2006;32:937-40.

20. Sacchini V, Pinotti JA, Barros AC, Luini A, Pluchinotta A, Pinotti M, et al. Nipple-sparing mastectomy for breast cancer and risk reduction: oncologic or technical problem? J Am Coll Surg 2006;203:704-14.

21. Donker M, van Tienhoven G, Straver ME, Meijnen P, van de Velde CJ, Mansel RE, et al. Radiotherapy or surgery of the axilla after a positive sentinel node in breast cancer (EORTC 10981-22023 AMAROS): a randomised, multicentre, open-label, phase 3 non-inferiority trial. Lancet Oncol 2014;15: 1303-10.

22. Poodt IGM, Spronk PER, Vugts G, van Dalen T, Peeters MTFDV, Rots ML, et al. Trends on axillary surgery in nondistant metastatic breast cancer patients treated between 2011 and 2015: a Dutch population-based study in the ACOSOG-Z0011 and AMAROS era. Ann Surg 2018;268:1084-90.

23. Giuliano AE, Hunt KK, Ballman KV, Beitsch PD, Whitworth PW, Blumencranz PW, et al. Axillary dissection vs no axillary dissection in women with invasive breast cancer and sentinel node metastasis: a randomized clinical trial. JAMA 2011;305: 569-75.

24. Giuliano AE, Ballman KV, McCall L, Beitsch PD, Brennan MB, Kelemen PR, et al. Effect of axillary dissection vs no axillary dissection on 10-year overall survival among women with invasive breast cancer and sentinel node metastasis: the ACOSOG Z0011 (Alliance) randomized clinical trial. JAMA 2017;318:918-26.

25. Ak 1 nc 1 M, Bulut SP, Erözgen F, Gürbüzel M, Gülşen G, Kocakuşak A, et al. Predictive value of fine needle aspiration biopsy of axillary lymph nodes in preoperative breast cancer 
staging. Ulus Cerrahi Derg 2016;32:191-6.

26. Ganott MA, Zuley ML, Abrams GS, Lu AH, Kelly AE, Sumkin JH, et al. Ultrasound guided core biopsy versus fine needle aspiration for evaluation of axillary lymphadenopathy in patients with breast cancer. ISRN Oncol 2014;2014:703160.

27. Nathanson SD. Ultrasound-guided core needle biopsy of axillary lymph nodes in breast cancer. J Am Coll Surg 2012;214: 871-2.

28. Balasubramanian I, Fleming CA, Corrigan MA, Redmond HP, Kerin MJ, Lowery AJ. Meta-analysis of the diagnostic accuracy of ultrasound-guided fine-needle aspiration and core needle biopsy in diagnosing axillary lymph node metastasis. Br J Surg 2018;105:1244-53.

29. Solon JG, Power C, Al-Azawi D, Duke D, Hill AD. Ultrasoundguided core biopsy: an effective method of detecting axillary nodal metastases. J Am Coll Surg 2012;214:12-7.

30. Arslan G, Altintoprak KM, Yirgin IK, Atasoy MM, Celik L. Diagnostic accuracy of metastatic axillary lymph nodes in breast MRI. Springerplus 2016;5:735.

31. Kuijs VJ, Moossdorff M, Schipper RJ, Beets-Tan RG, Heuts EM,
Keymeulen KB, et al. The role of MRI in axillary lymph node imaging in breast cancer patients: a systematic review. Insights Imaging 2015;6:203-15.

32. Hyun SJ, Kim EK, Moon HJ, Yoon JH, Kim MJ. Preoperative axillary lymph node evaluation in breast cancer patients by breast magnetic resonance imaging (MRI): can breast MRI exclude advanced nodal disease? Eur Radiol 2016;26:3865-73.

33. Nakamura R, Yamamoto N, Miyaki T, Itami M, Shina N, Ohtsuka M. Impact of sentinel lymph node biopsy by ultrasound-guided core needle biopsy for patients with suspicious node positive breast cancer. Breast Cancer 2018;25:86-93.

34. Rao R, Lilley L, Andrews V, Radford L, Ulissey M. Axillary staging by percutaneous biopsy: sensitivity of fine-needle aspiration versus core needle biopsy. Ann Surg Oncol 2009;16: 1170-5.

35. Ahn HS, Kim SM, Jang M, Yun BL, Kim SW, Kang E, et al. Comparison of sonography with sonographically guided fine-needle aspiration biopsy and core-needle biopsy for initial axillary staging of breast cancer. J Ultrasound Med 2013;32:2177-84. 